\title{
A stochastic approach to 'dynamic-demand' refrigerator control
}

\author{
D. Angeli and P.A. Kountouriotis* \\ EEE Department, Imperial College, London, SW7 2AZ, UK
}

\begin{abstract}
Dynamic demand management is a very promising research direction for improving power system resilience. This paper considers the problem of managing power consumption by means of "smart" thermostatic control of domestic refrigerators. In this approach, the operating temperature of these appliances, and thus their energy consumption, is modified dynamically, within a safe range, in response to mains frequency fluctuations. Previous research has highlighted the potential of this idea for responding to sudden power plant outages. However, deterministic control schemes have proved inadequate as individual appliances tend to "synchronize" with each other, leading to unacceptable levels of overshoot in energy demand, when they "recover" their steady-state operating cycles.

In this paper we design random controllers that are able to respond to sudden plant outages and which avoid the instability phenomena associated with other feedback strategies. Stochasticity is used to achieve desynchronization of individual refrigerators while keeping overall power consumption tightly regulated.
\end{abstract}

Keywords: Jump linear systems, dynamic demand management, power system stability, refrigerators, load balancing.

\footnotetext{
*Email: \{d.angeli,pk201\}@imperial.ac.uk, Phone: +44(0)2075946281 Fax: +44(0)2075946282
} 


\section{Introduction}

Dynamic demand management is a very promising research direction for improving power system resilience. In a power grid, the system frequency (mains frequency) is an indicator of the balance between demand (load) and supply (generation), with the nominal frequency of $50 \mathrm{~Hz}$ corresponding to perfect balance between the two. When demand levels exceed the available supply, the frequency drops below $50 \mathrm{~Hz}$, while in the case of excess (with respect to system load) generation, the frequency rises above $50 \mathrm{~Hz}$. As a result, system frequency continuously fluctuates around the nominal level, and the system operator ensures that the balance between demand and supply is continuously maintained, stabilizing the frequency within narrow bands around $50 \mathrm{~Hz}$, by regulating the available supply.

In order for such (supply) regulation to be possible, however, it is required that 'frequency response services', as well as sufficient reserves, are included in the system ${ }^{1}$. This is essential not only for instantaneous frequency balancing, but, more importantly, for the ability to respond to sudden power plant failures, which would otherwise lead to severe blackouts.

From an economic perspective, frequency response services and reserve power are costly, and any method which manages to reduce the magnitude of these services, without sacrificing system stability, is of significant importance [8]. In recent years, research has been initiated on the possibility of using frequency responsive loads, commonly referred to as "dynamic demand control", so as to reduce the amount of frequency response and reserve services that are required, potentially leading to significant reductions in overall system costs.

In this paper, we consider the problem of managing power demand by means of "smart" thermostatic control of domestic refrigerators. In this approach, the operating temperature

\footnotetext{
${ }^{1}$ Frequency response services are provided by synchronized generators, running only part-loaded (and hence not at maximum efficiency), as well as from industrial customers [1]. Reserve power is identified with slower, part-loaded plants, and generation units that can start producing at short notice.
} 
of these appliances, and thus their energy consumption, is modified dynamically, within a safe range, in response to mains frequency fluctuations. Previous research $([8],[3],[1])$ has shown that this is an effective way to respond to sudden power plant outages, reducing the cost of reserve power required to deal with such events. The feasibility of the approach for demand management stems from the large number of domestic refrigerators that are in use (around 40 million appliances are estimated to operate in the UK [1]). In addition, similar control schemes can be employed for any other types of appliances, both domestic and industrial, that exhibit energy storage in the form of heat, such as freezers, water heaters, etc. [8], greatly expanding the potential applications.

More closely, [8] and [3] investigate the potential of dynamic demand control of domestic refrigerators, when the thermostat's temperature thresholds (and, thus, the duty cycle of appliances) are varied as linear functions of mains frequency deviation from its nominal value, while they also perform an assessment of the control method in scenarios with significant supply variability, due to power generated by wind turbines. In both cases, their results demonstrate that the amount of standing reserve required by the power system can be safely reduced. A similar approach is followed in [1], where the economic impacts of such control strategies are also quantified depending on the types of generation units in the system (nuclear plants, coal plants, combined cycle gas turbine plants, etc.).

Simple feedback schemes, however, such as those employed in [8] and [3], in which the operating temperature is varied in a linear fashion with respect to mains frequency deviations, can prove inadequate in achieving desired performance, as individual appliances tend to "synchronize" with each other, leading to unacceptable levels of overshoot in energy demand, when they "recover" their steady-state operating temperatures. The appearance of such phenomena can be slow, but they do ultimately lead to unstable oscillations in the frequency of the overall system.

The problem of dynamic demand management is also addressed in [10], in the context 
of (centralized) model predictive control (MPC). In this case, the appliances are assumed to be connected to a communications network, and are able to receive and execute commands that are generated by a central processing node. The approach is applied to problems in which there is considerable supply variation due to the significant employment of alternative energy sources (e.g. wind and photovoltaic). Non-anticipated events such as generator failures are not explicitly addressed. As expected, the closed loop behaviour is far superior to that corresponding to the simpler schemes of [8] and [3], but the prospects of immediate utilization of such ideas are not enhanced, not least by the extra costs that would be required for widespread implementation.

In contrast to [10], we adopt a framework in which there is no communication between the controlled devices, and so each device has to act in an autonomous setting. While this is a severe constraint, and complicates the problem, we note that the quantity of interest is the temperature distribution of the whole population of appliances at each particular time point. We therefore pose the problem in a probabilistic framework, in which we try to find control schemes that steer the probability densities involved towards desired distributions. The advantage of this approach is that it greatly reduces the dimensionality of the original problem, while it allows for simple, yet successful solutions.

A viable control scheme in this setting, is the replacement of classical hysterisis-based controllers with controls that randomly jump between the "on" and "off" states of the appliances. Careful selection of the jump propensities allows for the decentralized control of individual appliances' duty cycles (and, therefore, power consumption), while, during "recovery", the "population" of refrigerators is sufficiently diversified (mixed) with respect to temperature, thereby avoiding undesirable overshoot phenomena.

The probabilistic description of the problem allows for the derivation of closed-form expressions for the first two moments of the temperature distributions involved, in terms of the aforementioned jump propensities, so that the latter can be selected in order to 
control these quantities of interest. The resulting closed loop system can be shown to exhibit properties of local asymptotic stability, regardless of parameter values, as well as boundedness of solutions for all initial conditions.

The performance of the proposed controllers is assessed via simulations, when coupled with a simple model of the power grid. Initial results verify the theoretical underpinnings of our approach, and clearly illustrate the robustness of the method when compared to earlier approaches.

The rest of the paper is organized as follows. Section 2 elaborates the mathematical results enabling the proposed solution. A model of refrigerators is outlined in Section 2.1, with the corresponding analysis shown in Section 2.2. The interconnected system and the random controller are described in Section 3, while Section 4 proceeds with a stability analysis of the closed loop system. Results of simulation studies are shown in Section 5. Conclusions can be found in Section 6, while the instability of deterministic control schemes is discussed in the Appendix.

\section{A stochastic approach to refrigerator control}

\subsection{Refrigerator modeling}

For the purposes of deriving a random control algorithm, refrigerators are modelled as Markov jump linear systems $[2,6]$, or to be more precise jump affine systems. Roughly speaking these are switched affine systems whose driving signal is the stochastic process associated to a finite Markov chain. In particular, in this context, we consider Markov chains with two states only, an $\mathrm{OFF}$ and an ON state, and transition probability rates between them which are denoted by $\lambda_{1}$ and $\lambda_{2}$ respectively. It is customary to graphically represent such systems as done in Fig. 1. Letting $T(t)$ denote the temperature of a single appliance at time $t$, its evolution in each of the two states is described by an affine, first- 
order ordinary differential equation, as

$$
\begin{array}{cc}
\dot{T}(t)=-\alpha\left(T(t)-T_{O N}\right) & \text { when } \mathrm{ON} \\
\dot{T}(t)=-\alpha\left(T(t)-T_{O F F}\right) & \text { when } \mathrm{OFF}
\end{array}
$$

In (1) $T_{O F F}$ and $T_{O N}$ denote respectively the ambient temperature and the steady-state temperature reached by a refrigerator which is always ON. The positive coefficient $\alpha$ is a thermal dispersion coefficient.

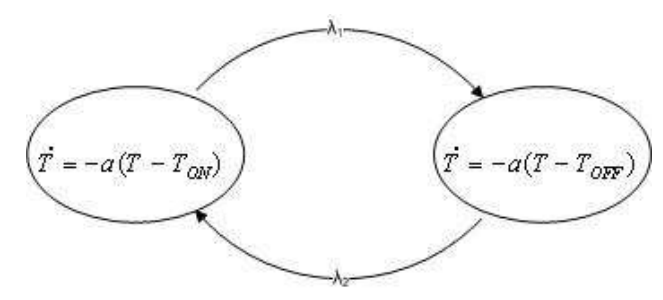

Figure 1: Markov chain illustration

We also use $\pi_{O N}(t)$ and $\pi_{O F F}(t)$ to denote the probability of a single refrigerator being in the ON and OFF state respectively. Obviously, $\pi_{O N}(t)+\pi_{O F F}(t)=1$ for all times $t$. The equations governing the evolution in time of such probabilities are therefore given as:

$$
\begin{aligned}
\dot{\pi}_{O N}(t) & =-\lambda_{1} \pi_{O N}(t)+\lambda_{2} \pi_{O F F}(t) \\
\dot{\pi}_{O F F}(t) & =-\lambda_{2} \pi_{O F F}(t)+\lambda_{1} \pi_{O N}(t) .
\end{aligned}
$$

Due to ergodicity of the underlying Markov Chain, the vector $\left(\pi_{O N}(t), \pi_{O F F}(t)\right)^{T}$ for each given pair $\left(\lambda_{1}, \lambda_{2}\right) \in(0,+\infty)^{2}$, converges to a unique stationary distribution $\left(\bar{\pi}_{O N}, \bar{\pi}_{O F F}\right)^{T}$. In particular, $\bar{\pi}_{O N}$ also represents the average duty cycle of a single appliance. It is straightforward to see that:

$$
\bar{\pi}_{O N}=\frac{\lambda_{2}}{\lambda_{1}+\lambda_{2}} \quad \bar{\pi}_{O F F}=\frac{\lambda_{1}}{\lambda_{1}+\lambda_{2}} .
$$


Due to the low-dimensionality of the Markov chain, explicit expressions for the transient probability distributions can be computed as follows

$$
\begin{aligned}
\pi_{O N}(t) & =e^{-\left(\lambda_{1}+\lambda_{2}\right) t} \pi_{O N}(0)+\left(1-e^{-\left(\lambda_{1}+\lambda_{2}\right) t}\right) \bar{\pi}_{O N} \\
\pi_{O F F}(t) & =e^{-\left(\lambda_{1}+\lambda_{2}\right) t} \pi_{O F F}(0)+\left(1-e^{-\left(\lambda_{1}+\lambda_{2}\right) t}\right) \bar{\pi}_{O F F} .
\end{aligned}
$$

It is worth pointing out that these are both monotone functions of time. Therefore, if parameters $\lambda_{1}$ and $\lambda_{2}$ undergo a step change, the corresponding probability distributions will evolve monotonically to their new steady-state value. As power absorption of a population of appliances is proportional to the fraction of them which are in the ON state, monotonicity of $\pi_{O N}(t)$ is a first important indication of the absence of undesired overshoots in power absorption.

\subsection{Open loop behaviour: analytical results}

As our goal is to regulate the overall behaviour of a population of refrigerators, it is convenient to derive formulae that describe the time-evolution of the probability distribution of temperatures of a single appliance, and expressions for the associated first two moments. To this end, let $\rho_{+}(t, T)$ and $\rho_{-}(t, T)$ denote the unnormalized pdf of the temperature of a device in the $\mathrm{ON}$ and $\mathrm{OFF}$ state respectively, at time $t$. In particular note that

$$
\pi_{O N}(t)=\int_{-\infty}^{+\infty} \rho_{+}(t, T) d T, \quad \pi_{O F F}(t)=\int_{-\infty}^{+\infty} \rho_{-}(t, T) d T
$$

so that

$$
\int_{-\infty}^{+\infty} \rho_{+}(t, T) d T+\int_{-\infty}^{+\infty} \rho_{-}(t, T) d T=1
$$


These (unnormalized) temperature distributions satisfy a form of Kolmogorov's forward equation, such that

$$
\begin{gathered}
\frac{\partial \rho_{+}}{\partial t}=\alpha\left(T-T_{O N}\right) \frac{\partial \rho_{+}}{\partial T}+\left(\alpha-\lambda_{1}\right) \rho_{+}+\lambda_{2} \rho_{-} \\
\frac{\partial \rho_{-}}{\partial t}=\alpha\left(T-T_{O F F}\right) \frac{\partial \rho_{-}}{\partial T}+\left(\alpha-\lambda_{2}\right) \rho_{-}+\lambda_{1} \rho_{+} .
\end{gathered}
$$

Even though equations (4) admit no closed-form solution it is possible, thanks to the affine nature of the underlying dynamics, to obtain ODEs that describe the evolution of the first two moments associated with these distributions, as well as asymptotic (steady-state) values for these moments. To this end, we define $T_{+}(t)$ and $T_{-}(t)$ as

$$
\begin{aligned}
T_{+}(t) & =\int_{-\infty}^{+\infty} T \rho_{+}(t, T) d T \\
T_{-}(t) & =\int_{-\infty}^{+\infty} T \rho_{-}(t, T) d T
\end{aligned}
$$

so that $E[T(t)]=T_{+}(t)+T_{-}(t)$. Differentiating the previous formulae with respect to time, and using (4), we obtain differential equations for the time evolution of these quantities, as follows:

$$
\begin{aligned}
& \dot{T}_{+}=-\left(\alpha+\lambda_{1}\right) T_{+}+\lambda_{2} T_{-}+\alpha \pi_{O N} T_{O N} \\
& \dot{T}_{-}=-\left(\alpha+\lambda_{2}\right) T_{-}+\lambda_{1} T_{+}+\alpha \pi_{O F F} T_{O F F} .
\end{aligned}
$$

It then follows that the expected temperature $E[T(t)]$ satisfies the differential equation

$$
E[\dot{T}(t)]=-\alpha\left(E[T(t)]-\pi_{O N} T_{O N}-\pi_{O F F} T_{O F F}\right) .
$$

Taking into account the steady-state values of $\pi_{O N}$ and $\pi_{O F F}$ given in (3), the expected 
value of $T$ converges asymptotically to

$$
E[T(\infty)]=\bar{\pi}_{O F F} T_{O F F}+\bar{\pi}_{O N} T_{O N}=\frac{\lambda_{1}}{\lambda_{1}+\lambda_{2}} T_{O F F}+\frac{\lambda_{2}}{\lambda_{1}+\lambda_{2}} T_{O N} .
$$

Now consider the variance $v(t)=\operatorname{var}[T(t)]$ of $T$. This is given as

$$
\begin{aligned}
v(t) & =\int_{-\infty}^{+\infty}(T-E[T])^{2}\left(\rho_{+}(t, T)+\rho_{-}(t, T)\right) d T \\
& =\int_{-\infty}^{+\infty} T^{2}\left(\rho_{+}(t, T)+\rho_{-}(t, T)\right) d T-(E[T])^{2} .
\end{aligned}
$$

Differentiating the above with respect to $t$, and using (4), yields

$$
\dot{v}(t)=-2\left[\alpha\left[v(t)+(E[T])^{2}-T_{O N} T_{+}-T_{O F F} T_{-}\right]+E[T] E[T]\right] .
$$

Overall, the first and second population's moments are governed by the following blocktriangular set of ODEs:

$$
\begin{aligned}
\dot{\pi}_{O N}= & -\lambda_{1} \pi_{O N}+\lambda_{2} \pi_{O F F} \\
\dot{\pi}_{O F F}= & -\lambda_{2} \pi_{O F F}+\lambda_{1} \pi_{O N} \\
\dot{T}_{+}= & -\left(\alpha+\lambda_{1}\right) T_{+}+\lambda_{2} T_{-}+\alpha \pi_{O N} T_{O N} \\
\dot{T}_{-}= & -\left(\alpha+\lambda_{2}\right) T_{-}+\lambda_{1} T_{+}+\alpha \pi_{O F F} T_{O F F} \\
\dot{v}= & -2\left[\alpha\left[v+\left(T_{+}+T_{-}\right)^{2}-T_{O N} T_{+}-T_{O F F} T_{-}\right]+\right. \\
& \left.-\left(T_{+}+T_{-}\right) \alpha\left(T_{+}+T_{-}-\pi_{O N} T_{O N}-\pi_{O F F} T_{O F F}\right)\right] .
\end{aligned}
$$

Due to the cascaded structure of this system and linearity of its diagonal terms, it is easy to see that the system is globally asymptotically convergent. In particular, the asymptotic 
value for the variance equals:

$$
\bar{v}=\left(T_{O N}-T_{O F F}\right)^{2} \frac{\alpha \lambda_{1} \lambda_{2}}{\left(\lambda_{1}+\lambda_{2}\right)^{2}\left(\alpha+\lambda_{1}+\lambda_{2}\right)} .
$$

The formulae derived so far will be useful also for the derivation of a control strategy which is developed in the next Section.

\section{The interconnected system}

The power grid is modeled by a 3rd order linear system [4]

$$
\left(\begin{array}{c}
\Delta \dot{P}_{v} \\
\Delta \dot{P}_{m} \\
\dot{\Delta \omega}
\end{array}\right)=\left(\begin{array}{ccc}
-1 / T_{g} & 0 & -1 /\left(R_{e q} T_{g}\right) \\
1 / T_{t} & -1 / T_{t} & 0 \\
0 & 1 / M & -D / M
\end{array}\right)\left(\begin{array}{c}
\Delta P_{v} \\
\Delta P_{m} \\
\Delta \omega
\end{array}\right)+\left(\begin{array}{c}
0 \\
0 \\
-1 / M
\end{array}\right) \frac{\left(\Delta P_{L}-\Delta P_{f}\right)}{P_{\text {tot }}}
$$

corresponding to the block diagram shown in Figure 2. Exact values for the parameters in (14) are given in Section 5. Notice that (14) is expressed in a 'per-unit' basis ([1]), so that $\Delta f=50 \times \Delta \omega$.

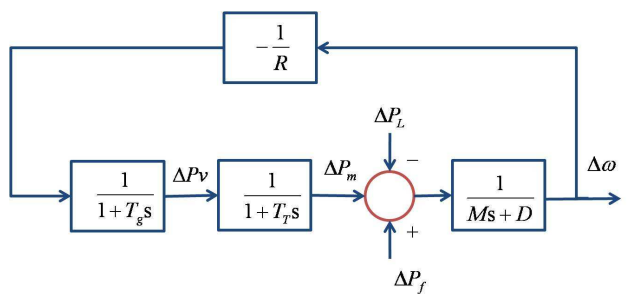

Figure 2: Model of power grid

The appliances load the grid at the summing junction via the variable $\Delta P_{f}$, which represents the deviation in overall power (consumed by all the appliances that are connected to the network) from their nominal consumption level. The additional variable $\Delta P_{L}$ is used 
so as to simulate a sudden loss of power in the system².

Standard refrigerator controllers operate on a hysteretic basis, in which two temperature levels $T_{\max }$ and $T_{\min }$ trigger the motor $\mathrm{ON}$ and $\mathrm{OFF}$ respectively. Initial attempts at dynamic demand refrigerator control $([8],[1])$ focused on dynamically adjusting these threshold levels, by imposing a linear dependence on mains frequency deviations, as

$$
\begin{aligned}
& \tilde{T}_{\text {max }}(t)=T_{\max }+K \Delta f(t) \\
& \tilde{T}_{\text {min }}(t)=T_{\text {min }}+K \Delta f(t),
\end{aligned}
$$

where $K$ is a constant of proportionality.

Even though such strategies are effective in the short-term (i.e. when for the first time a plant failure occurs), evidence is provided in the Appendix and Simulation Sections that they eventually lead to unstable overall behaviour of the closed-loop system. This phenomenon takes two different forms:

- long-term phase synchronization of refrigerators: indeed even non-identical population of refrigerators with duty cycles of comparable duration will tend to asymptotically synchronize their oscillations, giving rise to the so called phase-locking phenomenon, see for instance [7].

- uncontrolled modifications of the population's temperature distribution: the uniform in phase distribution that one expects of a population of randomly switched on utilities gets unpredictably modified by the occurence of load disturbances and leads to significant oscillations in power demand even in the medium term.

In the rest of this Section, we describe an alternative control strategy that avoids such instabilities.

\footnotetext{
${ }^{2}$ Note that a sudden power loss is equivalent to a sudden increase in demand, of the same magnitude.
} 


\subsection{Random control strategy}

In what follows, it is constructive to define the control variables $u_{1}$ and $u_{2}$ as

$$
u_{1}=\frac{\lambda_{1}}{\alpha} \quad \text { and } \quad u_{2}=\frac{\lambda_{2}}{\alpha}
$$

Now consider equations (10) and (13). In terms of the new variables, these can be rewritten as

$$
\begin{aligned}
E[T(\infty)] & =\frac{u_{1}}{u_{1}+u_{2}} T_{O F F}+\frac{u_{2}}{u_{1}+u_{2}} T_{O N} \\
\bar{v} & =\left(T_{O N}-T_{O F F}\right)^{2} \frac{u_{1} u_{2}}{\left(u_{1}+u_{2}\right)^{2}\left(1+u_{1}+u_{2}\right)}
\end{aligned}
$$

Note also that equation (3), which determines the duty cycle of each appliance as a function of the transition rates, can be rewritten as

$$
\bar{\pi}_{O N}=\frac{u_{2}}{u_{1}+u_{2}}
$$

Control of the appliances can be achieved via the selection of the transition rates $\lambda_{1}$ and $\lambda_{2}$ as functions of the grid frequency deviation $\left(f_{n o m}-f(t)\right)$. By fixing a desired value for the variance in operating temperatures, $v_{\text {des }}$ in (18), the transition rates $\lambda_{1}$ and $\lambda_{2}$ can be determined by postulating a desired average temperature, $T_{\text {des }}$, or a desired average duty cycle, $\pi_{\text {des }}$. In particular, if the latter is adopted, the following expressions are obtained by inverting the previous formulas:

$$
\begin{aligned}
& u_{1}=\left(\pi_{d e s}^{2}-\pi_{\text {des }}+v_{\text {des }} /\left(T_{O N}-T_{O F F}\right)^{2}\right)\left(\pi_{d e s}-1\right)\left(T_{O N}-T_{O F F}\right)^{2} / v_{d e s} \\
& u_{2}=-\pi_{\text {des }}\left(\pi_{\text {des }}^{2}-\pi_{\text {des }}+v_{\text {des }} /\left(T_{O N}-T_{O F F}\right)^{2}\right)\left(T_{O N}-T_{O F F}^{2}\right) / v_{d e s}
\end{aligned}
$$

Our decentralized control strategy is therefore to vary either $T_{d e s}$ or $\pi_{d e s}$ as linear functions 
of the frequency deviation:

$$
\begin{aligned}
& T_{\text {des }}(t)=T_{\text {nom }}+K_{T}\left(f_{\text {nom }}-f(t)\right) \\
& \pi_{\text {des }}(t)=\pi_{\text {nom }}+K_{\pi}\left(f_{\text {nom }}-f(t)\right),
\end{aligned}
$$

where $K_{T}$ and $K_{\pi}$ are proportionality constants, and $T_{n o m}$ and $\pi_{n o m}$ are the nominal values of the average temperature and the (corresponding) duty cycle when there is no frequency deviation in the grid $(\Delta f=0)$.

In the simulations shown in Section $5, \pi_{d e s}$ was chosen as the reference variable, as it led to faster responses.

The control scheme described above results in a time-inhomogeneous Markov chain, with rate functions $\lambda_{1}(t)$ and $\lambda_{2}(t)$ which can be computed as functions of the instantaneous mains frequency $f(t)$, just by composing equations (20) and (22).

Individual appliances, then, will run the following simple algorithm:

- When device switches to ON mode:

1. Set $t_{0}=t$ and $\mathrm{r}=\mathrm{RND}$.

2. Start evaluating the integral $I(t)=\int_{t_{0}}^{t} \lambda_{1}(\tau) d \tau$.

3. Switch to $\mathrm{OFF}$ at time $t^{\prime}$, for which $I\left(t^{\prime}\right) \geq-\ln (r)$.

- When device switches to OFF mode:

1. Set $t_{0}=t$ and $\mathrm{r}=\mathrm{RND}$.

2. Start evaluating the integral $I(t)=\int_{t_{0}}^{t} \lambda_{2}(\tau) d \tau$.

3. Switch to OFF at time $t^{\prime}$, for which $I\left(t^{\prime}\right) \geq-\ln (r)$.

In the above, RND denotes a random number, uniformly distributed in the interval $[0,1]$. 
Note that the scheme is computationally simple, in that it only involves a random number generator and a standard quadrature routine.

\subsection{Algorithm variations}

Based on the random control strategy of Section 3.1, several 'hybrid' algorithms can be constructed.

For example, even though the random controller of the previous Section regulates the mean and variance of a refrigerator's temperature, it might be desirable in practical applications to introduce safety thresholds $T_{\max }$ and $T_{\min }$, which would serve to prohibit temperature excursions beyond 'safe' levels. This leads to a random controller with temperature constraints, according to which, if any of the $T_{\max }$ or $T_{\min }$ thresholds is exceeded, the appliance forcibly switches $\mathrm{ON}$ (or, respectively, OFF), overriding the random control action.

In addition, initial simulations indicated that, even though the proposed random controller performs overall better than simpler linear feedback schemes, the latter respond faster at the onset of a failure, where the initial frequency drop is very sharp. To cater for the slower response, the $T_{\min }$ 'safety' threshold can be made frequency dependent, resulting in a random controller with variable constraints, as follows:

$$
\bar{T}_{\min }=T_{\min }-K_{s} \Delta f
$$

\section{Stability analysis}

We now proceed to the stability analysis of a large population of refrigerators governed by the random algorithm described in Section 3.1 connected to the power supply network as modeled by equations (14). Our main result in this respect is stated below: 
Claim: the interconnection of a large population of identical refrigerators, regulated according the random algorithm described above, yields a locally asymptotically stable closed-loop system (assuming the model (14)) regardless of parameters values and control gains.

To substantiate our claim we first derive a closed-loop mathematical model of this large scale system. To this end, it is useful to recall that equations (4) carry both a stochastic and a deterministic interpretation. From the stochastic point of view they describe the evolution of probability distributions of a continuous time random process (a single randomly regulated refrigerator). From the deterministic point of view, the quantity $\int_{\theta_{1}}^{\theta_{2}} \rho_{S}(t, \theta) d \theta$ can be seen as the fraction of refrigerators in state $S$ that at any given time $t$ have temperature in the interval $\left[\theta_{1}, \theta_{2}\right]$ provided a large population of identical refrigerators is assumed each of which updates its state according to the previously illustrated algorithm. It is worth pointing out that equations (4) keep their validity also if $\lambda_{1}$ and $\lambda_{2}$ are time-varying inputs. Moreover, the deterministic interpretation just mentioned allows to derive the closed-loop system simply by juxtaposition of equations (4) with the power-network model (14):

$$
\begin{aligned}
\frac{\partial \rho_{+}}{\partial t} & =\alpha\left[T-T_{O N}\right] \frac{\partial \rho_{+}}{\partial T}+\left(\alpha-\lambda_{1}(f)\right) \rho_{+}+\lambda_{2}(f) \rho_{-} \\
\frac{\partial \rho_{-}}{\partial t} & =\alpha\left[T-T_{O F F}\right] \frac{\partial \rho_{-}}{\partial T}+\left(\alpha-\lambda_{2}(f)\right) \rho_{-}+\lambda_{1}(f) \rho_{+} \cdot \\
f & =G(s)\left[L_{n}-K \pi_{O N}\right]
\end{aligned}
$$

where we denoted $G(s)$ the transfer function from $\Delta P_{f}$ to $\Delta f$. Notice that the load due to refrigerators is proportional trough the gain $K$ to the fraction of fridges which are in the ON state $\left(\pi_{O N}\right)$. The input $L_{n}$ is instead the nominal load. While equations (23) 


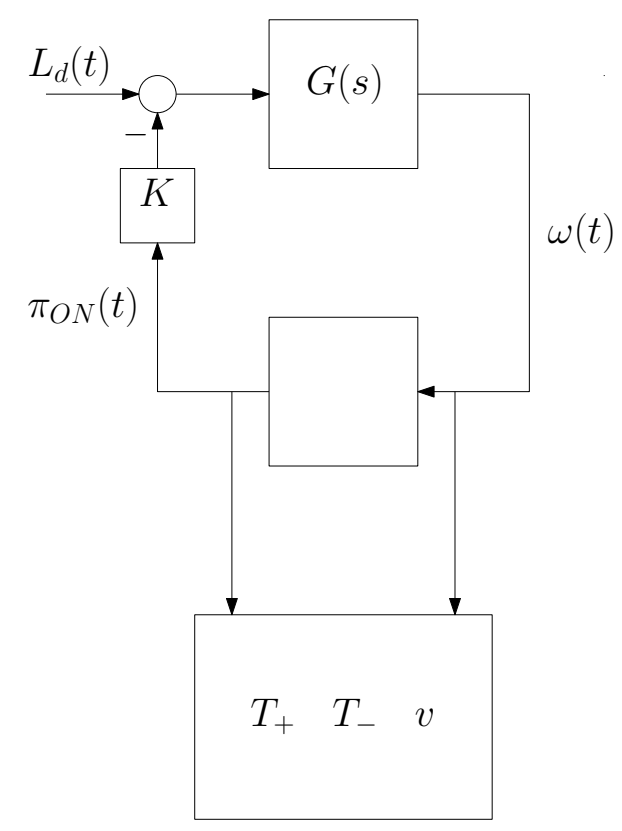

Figure 3: Closed-loop system

are infinite-dimensional, their momenta evolve according to a finite dimensional nonlinear system of equations. In particular:

$$
\begin{aligned}
\dot{\pi}_{O N} & =-\lambda_{1}(f) \pi_{O N}+\lambda_{2}(f) \pi_{O F F} \\
\dot{\pi}_{O F F} & =-\lambda_{2}(f) \pi_{O F F}+\lambda_{1}(f) \pi_{O N} \\
f & =G(s)\left[L_{n}-K \pi_{O N}\right] .
\end{aligned}
$$

The equations for $T_{+}, T_{-}$and $v$ are as in (12). Notice that the overall system exhibits a cascaded structure with the $\pi_{O N^{-}} f$ feedback loop forcing the remaining variables $T_{-}, T_{+}$ and $v$, see Fig. 3. Notice that we may exploit the conservation law: $\pi_{O N}(t)+\pi_{O F F}(t)=1$ in order to write the $\pi_{O N}$ subsystem as a scalar nonlinear system:

$$
\begin{aligned}
\dot{\pi}_{O N} & =-\left[\lambda_{1}(f)+\lambda_{2}(f)\right] \pi_{O N}+\lambda_{2}(f), \\
f & =G(s)\left[L_{n}-K \pi_{O N}\right]
\end{aligned}
$$


It is convenient to denote the function $f_{O N}(\pi, f)$ :

$$
f_{O N}(\pi, f):=-\left[\lambda_{1}(f)+\lambda_{2}(f)\right] \pi+\lambda_{2}(f) .
$$

The nonlinear switching propensities $\lambda_{1}$ and $\lambda_{2}$ are designed so that:

$$
\forall f_{e} \exists \text { unique } \pi_{e} \in(0,1): f_{O N}\left(\pi_{e}, f_{e}\right)=0 \text {. }
$$

Moreover, for all $\pi \neq \pi_{e}$ in $[0,1]$ it holds

$$
\left(\pi-\pi_{e}\right) \cdot f_{O N}\left(\pi, f_{e}\right)<0
$$

which implies global asymptotic stability of the equilibrium $\pi_{e}$ for the constant input $f_{e}$. Linearization around such equilibrium yields the equations of a first-order asymptotically stable linear system of positive DC gain. This is a strictly passive system, (see for instance [5]). Due to passivity of $G(s)$ and the passivity theorem the equilibrium point of (25) is locally asymptotically stable regardless of parameters values. Moreover, so are also the equations involving higher-order momenta due to their cascaded structure. While global asymptotic stability of the closed-loop system appears difficult to prove, boundedness of $\pi_{O N}$ (always in $[0,1]$ ) and BIBO stability of $G(s)$ allow to conclude boundedness of solutions of the closed-loop system (25) for all initial conditions. By a standard analysis based on Input-to-State stability of cascades, (see for instance [9] ), it is then possible to show boundedness of first and second momenta of $\rho$ for all initial conditions. 


\section{Simulation results}

In this section, we present preliminary results on the performance of the random controller and its variations, outlined in Sections 3.1 and 3.2, and compare with the deterministic controller of [8], when these algorithms are employed to control a population of 40 million refrigerators ( $90 \%$ of which is assumed to be of the 'dynamic demand' type), connected to a power supply network as shown in Section 3.

The parameters of the power grid model (14) were set to the values used in [1], and are given in Table 1. Two different scenarios were considered, differing in the amount of total power being supplied by the network, so as to highlight the effects of the refrigerators in the closed loop system.

\begin{tabular}{|c|c|c|c|c|c|}
\hline$T_{g}$ & $T_{t}$ & $R_{e q}$ & $M$ & $D$ & $P_{t o t}(\mathrm{GW})$ \\
\hline 0.2 & 50 & 0.177 & 6.7 & 1 & $(\mathrm{a}) 55,(\mathrm{~b}) 25$ \\
\hline
\end{tabular}

Table 1: Grid parameter values

The nominal parameters in the refrigerator model (1), namely $\alpha, T_{O N}$, and $T_{O F F}$, were set to $2.4 \times 10^{-4},-38.3$ and 20 , respectively, corresponding to the cycle shown in Figure 4, and a nominal duty cycle of approximately 25\%. Both 'dynamic demand' and 'non-dynamic demand' (i.e. conventional) appliances were simulated, with 1000 appliances in each set, while the total power consumption of the two sets was scaled up so as to approximate the total of 40 million appliances. The state and temperature of each simulated refrigerator were randomly initialized, while model parameters for each appliance $\left(\alpha, T_{O N}, T_{O F F}\right)$ were also randomly chosen from a $[-15 \%,+15 \%]$ uniform window around the nominal values previously stated. The parameters (controller gains, etc.) employed in the various control algorithms are collected ${ }^{3}$ in Table 2 .

The performance of the various algorithms was assessed in the case of a sudden loss of

\footnotetext{
${ }^{3}$ To ensure $0 \leq \pi_{O N} \leq 1$, a floor and ceiling value were introduced when calculating $\pi_{d e s}$ according to equation (22) in the random algorithms.
} 

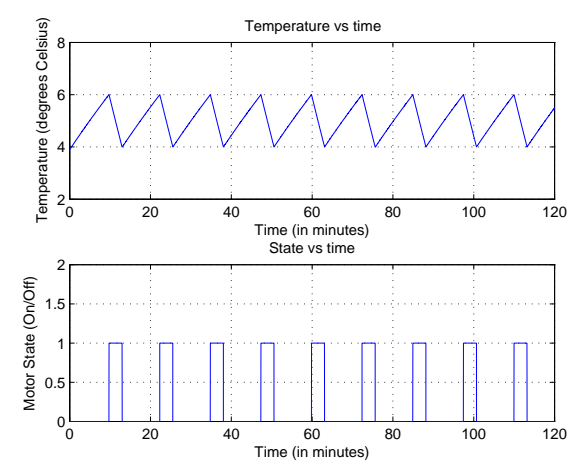

Figure 4: Fridge Cycle

\begin{tabular}{|c|c|}
\hline Hysteretic & $T_{\min }=4, T_{\max }=6$ \\
\hline Deterministic & $T_{\min }=4, T_{\max }=6, K=5$ \\
\hline Random & $K_{\pi}=5, T_{\text {des }}=5, v_{\text {des }}=1$ \\
\hline Constrained Random & $K_{\pi}=5, T_{\text {des }}=5, v_{\text {des }}=1, T_{\min }=1, T_{\max }=9$ \\
\hline Variable Constrained & $K_{\pi}=5, T_{\text {des }}=5, v_{\text {des }}=1, T_{\min }=1, T_{\max }=9, K_{s}=10$ \\
\hline
\end{tabular}

Table 2: Controller parameter values

$1.32 G W$ of power from the system, which was imposed by introducing a step increase in $P_{L}$ in equation (14). The duration of the loss was 15 minutes, after which the power recovered to the original levels in a ramp fashion, with the recovery period lasting 10 minutes (see Figure 5).

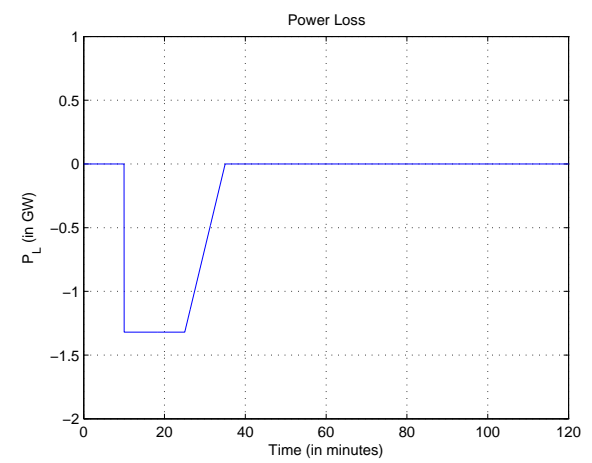

Figure 5: Power Loss

Figures 6(a) and 6(b) show the system frequency deviation $(\Delta f)$, the overall power 
consumed by refrigerators, and the average temprerature across the appliances as functions of time, for the two scenarios considered. The results demonstrate the superiority of the proposed control algorithm when compared to the deterministic control method of [8]; the closed loop is stable, while it also has the desired transient properties (see Figures 7(a) and 7(b) for a magnified version of the transient response).

The instability phenomena associated with the deterministic method (and which are elaborated upon in the Appendix) are highlighted in Figure 6, especially in cases where the total power consumed by the 'adaptive' refrigerators constitutes a significant fraction of the overall power demand in the system (Scenario II - Figure 6(b)). The random strategy does not suffer from such drawbacks.

In particular, the random controller (in all its variations) manages to stabilize the system's frequency at higher levels (i.e. smaller $\Delta f$ ) than the deterministic algorithm, without leading to undesirable overshoot in power consumption during the recovery phase, as is the case for the latter. A trade-off is identified between the absense of overshoot of the consumed power and the time required for the average temperature to recover its steady-state value, which is (significantly) longer for the random controller. The proposed scheme does not allow for the control of the recovery time, as the time constant in the expression for $E[T]$ (9) is equal to $1 / \alpha$, which is a device constant.

The 'variable constrained' version of the random controller responds as fast as the deterministic algorithm, while the introduction of 'safety' temperature thresholds does not adversely affect the closed loop performance.

\section{Conclusions}

A new algorithm for dynamic-demand control of refrigerator appliances has been presented and theoretically justified. The proposed algorithm adopts a probabilistc description of 

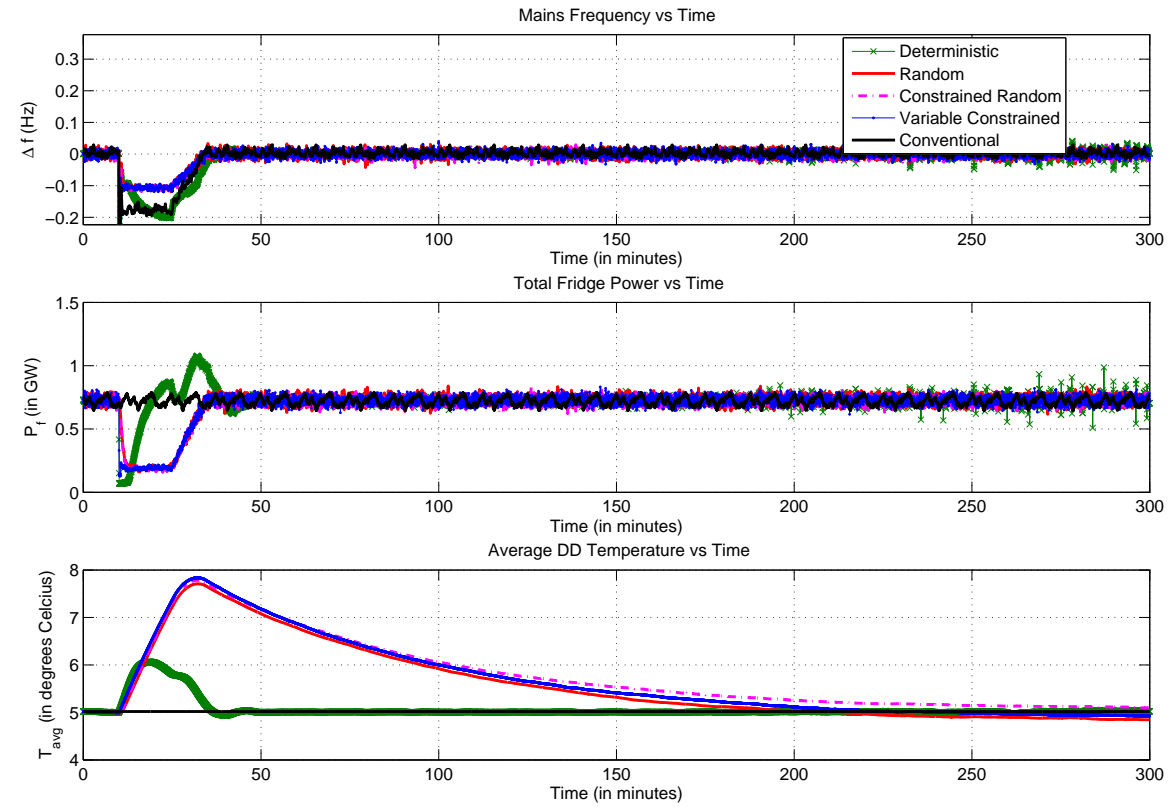

(a) Scenario I $\left(P_{t o t}=55 G W\right)$
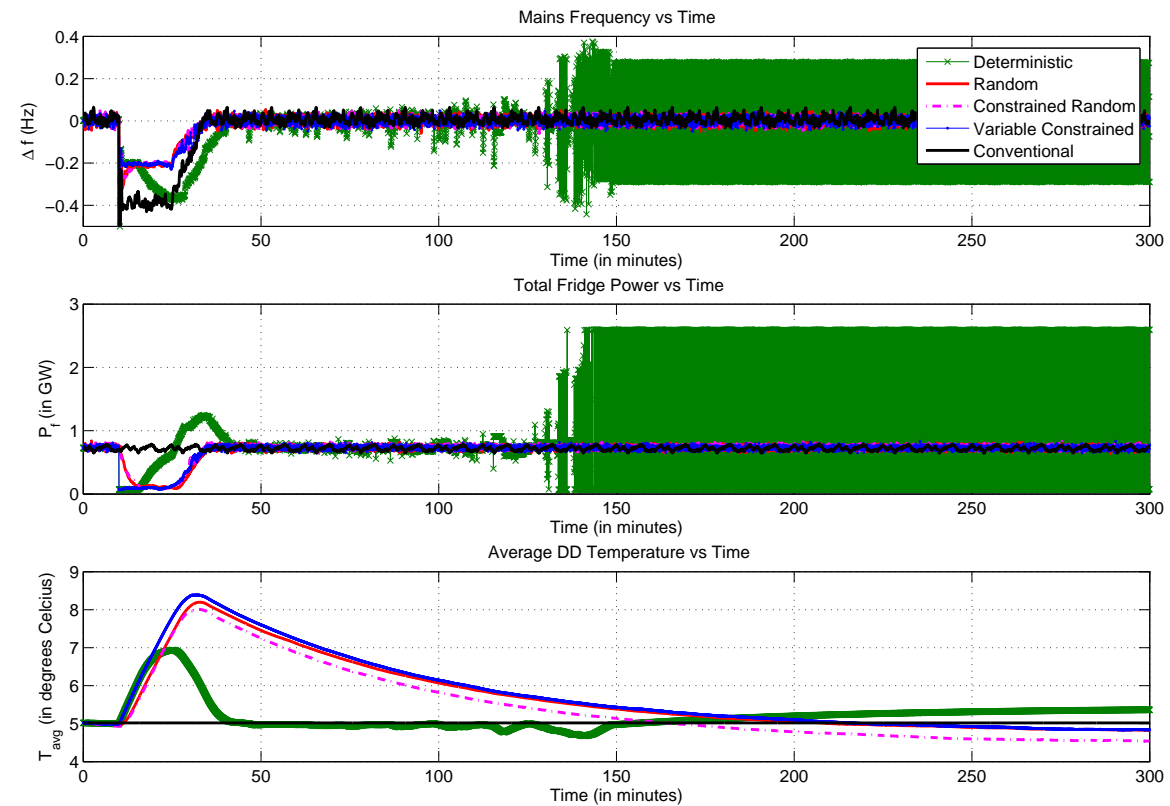

(b) Scenario II $\left(P_{t o t}=25 G W\right)$

Figure 6: Scenario I and II results 

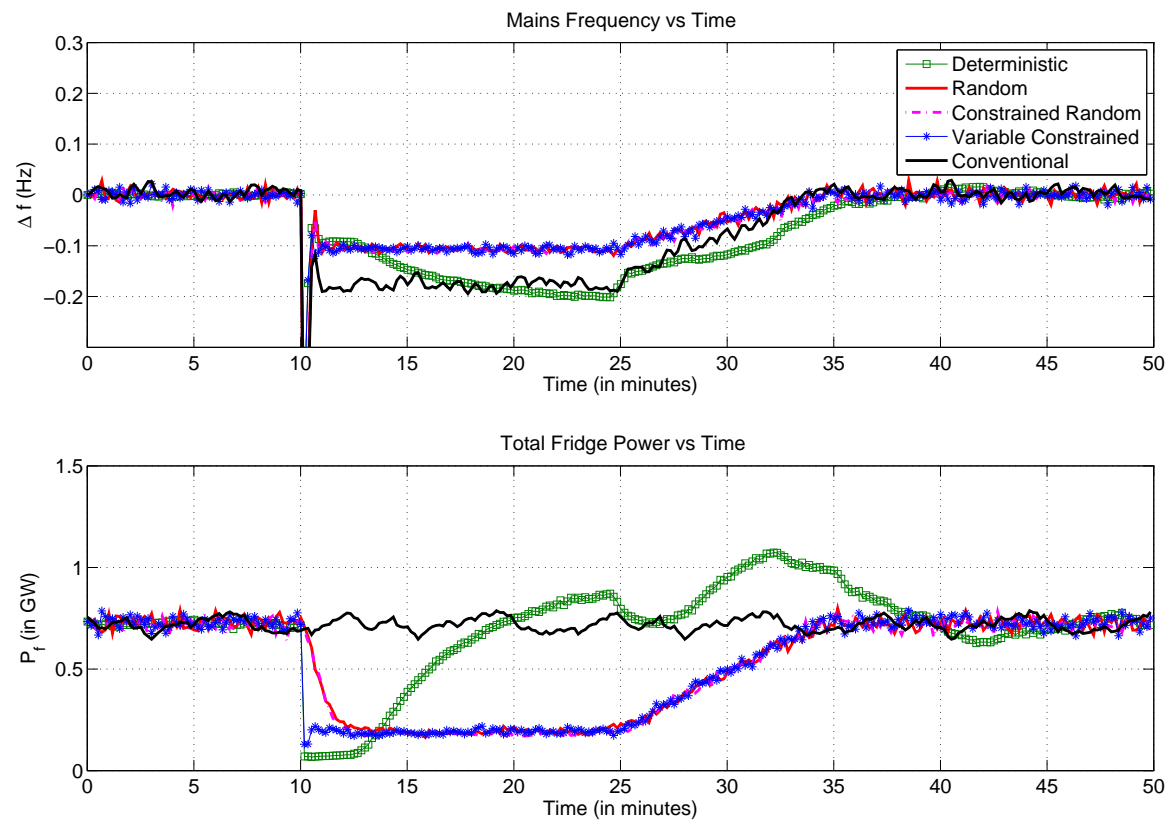

(a) Scenario I $\left(P_{t o t}=55 G W\right)$
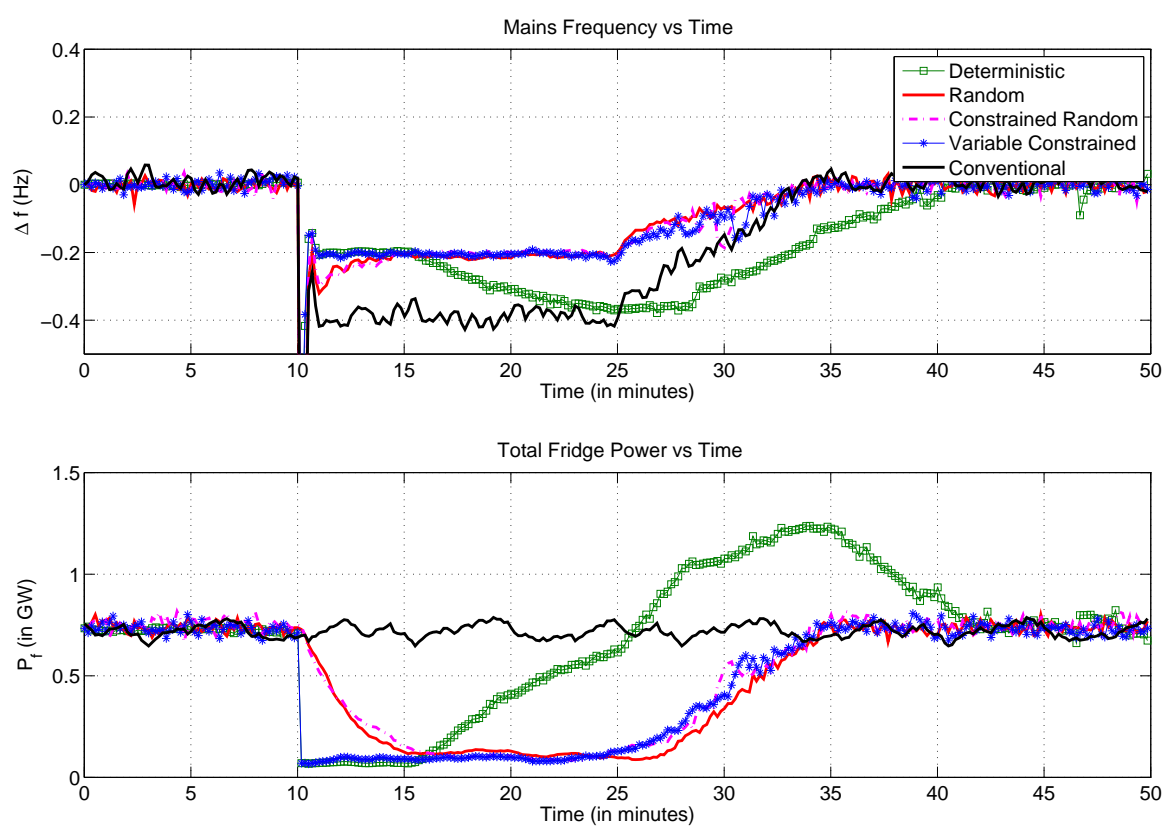

(b) Scenario II $\left(P_{t o t}=25 G W\right)$

Figure 7: Scenario I and II results (magnified) 
the problem, resulting in a relatively simple control scheme. Application of this control strategy ensures sufficient diversification (mixing) of the temperature across the controlled appliances, and does not lead to overshoot or instability phenomena associated with simpler deterministic schemes. The control scheme can also be applied to other kinds of devices that exhibit energy storage in the form of heat, such as freezers, water heaters, etc.. Similar ideas may also be exploited in other areas where the issue of node 'synchronization' potentially leads to problematic behaviour, such as in internet congestion studies.

Initial simulation results verify the theoretical underpinning of the proposed approach. The random controller is capable of maintaining the power system's frequency for a longer period of time, when compared to the deterministic scheme, and results in faster recovery at the end. Contrary to deterministic feedback, which breaks down in cases where the total average power consumed by refrigerators is large relative to the overall system demand, the random controller was shown to perform robustly.

Future work will focus on testing the approach in several scenarios of practical importance in the power systems industry, while an assessment of the potential economic benefits of the proposed method will also be undertaken.

\section{Acknoledgements}

This work is supported by the EPSRC grant "Control For Energy and Sustainability", grant reference EP/G066477/1.

\section{References}

[1] M. Aunedi, J.E. Ortega Calderon, V. Silva, P. Mitcheson, and G. Strbac, "Economic and Environmental Impact of Dynamic Demand," Centre for Sustain- 
able Electricity and Distributed Generation, November 2008, http://www.supergennetworks.org.uk/filebyid/50/file.pdf

[2] O.L. do Valle Costa, M. Dutra Fragoso and R. P. Marques, Discrete-time Markov jump linear systems. Springer-Verlag, London, 2005.

[3] D.G. Infield, J. Short, C. Horne, and L.L. Freris, "Potential for Domestic Dynamic Demand-Side Management in the UK," IEEE Power Engineering Society General Meeting 2007, pp.1-6, June 2007.

[4] D.I. Jones, "Dynamic system parameters for the National Grid," IEE Proceedings of Generation Transmission and Distribution, Vol. 152, No. 1, pp. 53-60, January 2005.

[5] R. Lozano, B. Brogliato, O. Egeland, and B. Maschke, Dissipative systems analysis and control. Springer-Verlag, London, 2000.

[6] M. Mariton, Jump linear systems in automatic control. Marcel Decker, 1990.

[7] A. Pikovsky, M. Rosenblum and J. Kurths, Synchronization: a universal concept in nonlinear sciences. Cambridge University Press, 2003.

[8] J.A. Short, D.G. Infield, and L.L. Freris, "Stabilization of Grid Frequency Through Dynamic Demand Control," IEEE Transactions on Power Systems, Vol. 22, No. 3, pp. 1284-1293, August 2007.

[9] E.D. Sontag, "Input to state stability: Basic concepts and results", In P. Nistri and G. Stefani, editors, Nonlinear and Optimal Control Theory, pp. 163220, Springer-Verlag, Berlin, 2007.

[10] M. Stadler, W. Krause, M. Sonnenschein, and U. Vogel, "Modelling and evaluation of control schemes for enhancing load shift of electricity demand for cooling devices," Elsevier Environmental Modelling and Software, Vol. 24, pp. 285-295, 2009. 


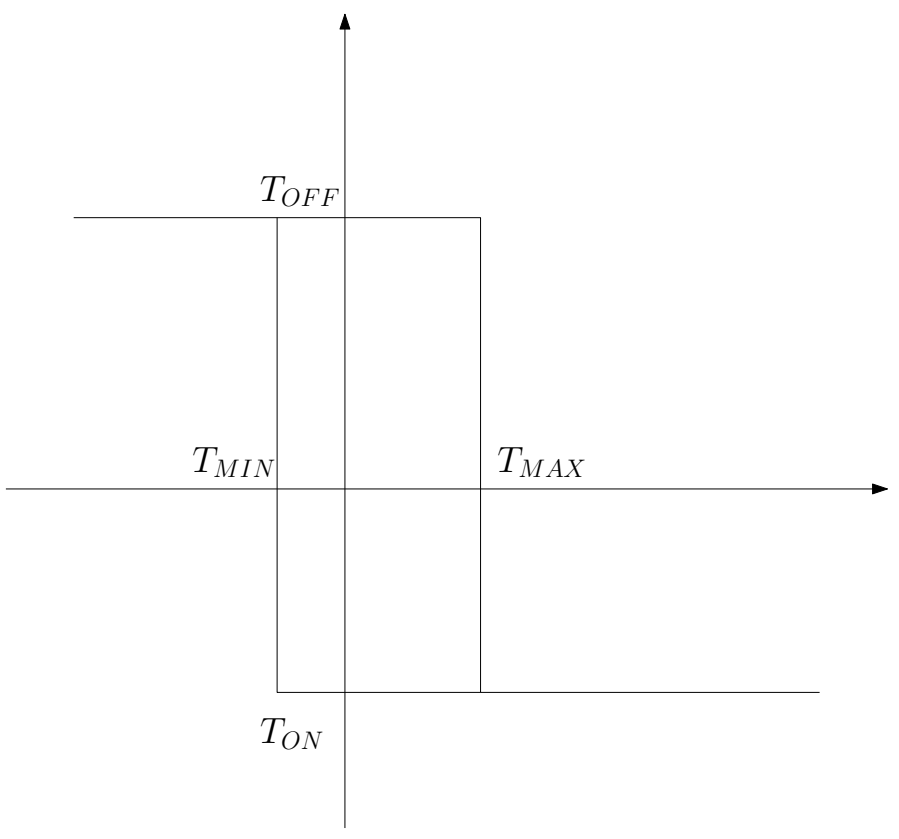

Figure 8: Relay nonlinearity

\section{A Phase-locking in deterministically regulated appli- ances}

The standard approach to temperature control in refrigerators is to determine the $\mathrm{ON} / \mathrm{OFF}$ state of the motor by means of a relay system. In this case equations governing temperature read:

$$
\dot{T}=-\alpha(T-H(T))
$$

where $H(T)$ is the hysteresis nonlinearity shown in Fig. 8. If $T_{M I N}$ and $T_{M A X}$ are held constant and $T_{O N}<T_{M I N}<T_{M A X}<T_{O F F}$, then the temperature $T$, converges in finite time to a periodic solution which has maximum and minimum at $T_{M I N}$ and $T_{M A X}$. This asymptotic solution $T_{s s}(t)$, has a period $\tau=t_{\text {on }}+t_{\text {off }}$ which can be explicitly computed 
by solving the two equations below:

$$
\begin{aligned}
T_{M I N}-T_{O N} & =e^{-\alpha \cdot t_{o n}}\left(T_{M A X}-T_{O N}\right) \\
T_{M A X}-T_{O F F} & =e^{-\alpha \cdot t_{o f f}}\left(T_{M I N}-T_{O F F}\right) .
\end{aligned}
$$

Indeed, assuming $T_{s s}(0)=T_{M A X}$ we have

$$
T_{s s}(t)=\left\{\begin{array}{cc}
e^{-\alpha t}\left(T_{M A X}-T_{O N}\right)+T_{O N} & \text { if } 0 \leq t \leq t_{o n} \\
e^{-\alpha\left(t-t_{o n}\right)}\left(T_{M I N}-T_{O F F}\right)+T_{O F F} & \text { if } t_{o n} \leq t \leq \tau
\end{array}\right.
$$

When a population of identical fridges is operated in parallel, the power absorption of the refrigerators asymptotically tends to a periodic function of period $\tau$. If the population is connected to a model of power network, this will induce a periodic fluctuation of mains frequency of the same period. For a uniform initial distribution of phases, however, the number of refrigerators in the $\mathrm{ON}$ state is approximately constant (and exactly so in the limiting case). This means that even a large population of fridges uniformly distributed with respect to phase only induces a neglegible fluctuation of mains frequency.

We analyze next what is the effect of periodically forcing equation (26) by defining the hysteresis threshold values $T_{M I N}$ and $T_{M A X}$ to be periodic functions of time. Let us assume:

$$
\begin{aligned}
& T_{O N}<T_{M I N}(t)<T_{M A X}(t)<T_{\text {OFF }} \quad \forall t \in \mathbb{R} \\
& T_{M I N}(t+\Upsilon)=T_{M I N}(t) \quad T_{M A X}(t+\Upsilon)=T_{M A X}(t) \quad \forall t \in \mathbb{R}
\end{aligned}
$$

for some positive number $\Upsilon$. In addition we may define:

$$
\begin{array}{cl}
\underline{T_{M I N}}=\min _{t \in \mathbb{R}} T_{M I N}(t) & \overline{T_{M I N}}=\max _{t \in \mathbb{R}} T_{M I N}(t) \\
\underline{T_{M A X}}=\min _{t \in \mathbb{R}} T_{M A X}(t) & \overline{T_{M A X}}=\max _{t \in \mathbb{R}} T_{M A X}(t) .
\end{array}
$$


Under such assumptions and with the notation introduced rough lower and upper-bounds to the duration of temperature oscillations for a forced refrigerator may be derived as follows:

$$
\begin{aligned}
& \tau_{M I N}=-\frac{1}{\alpha}\left[\log \left(\frac{\overline{T_{M I N}}-T_{O N}}{\underline{T_{M A X}}-T_{O N}}\right)+\log \left(\frac{T_{O F F}-\underline{T_{M A X}}}{\overline{T_{O F F}}-\overline{T_{M I N}}}\right)\right]
\end{aligned}
$$

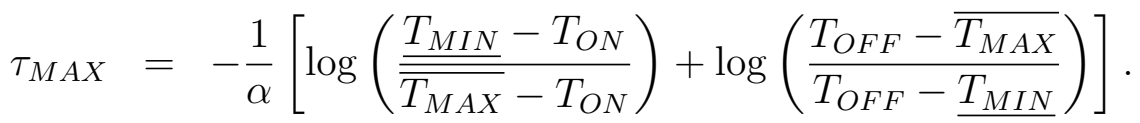

Tighter bounds are instead computed by solving:

$$
\begin{aligned}
\hat{\tau}_{M A X}= & \max _{\theta, t_{O N}, t_{O F F}} t_{O N}+t_{O F F} \\
& \text { subject to } \\
& T_{M I N}\left(\theta+t_{O N}\right)-T_{O N}=e^{-\alpha t_{O N}}\left[T_{M A X}(\theta)-T_{O N}\right] \\
& T_{M A X}\left(\theta+t_{O N}+t_{O F F}\right)-T_{O F F}=e^{-\alpha t_{O F F}}\left[T_{M I N}\left(\theta+t_{O N}\right)-T_{O F F}\right]
\end{aligned}
$$

and:

$$
\begin{aligned}
\hat{\tau}_{M I N}= & \min _{\theta, t_{O N}, t_{O F F}} t_{O N}+t_{O F F} \\
& \text { subject to } \\
& T_{M I N}\left(\theta+t_{O N}\right)-T_{O N}=e^{-\alpha t_{O N}}\left[T_{M A X}(\theta)-T_{O N}\right] \\
& T_{M A X}\left(\theta+t_{O N}+t_{O F F}\right)-T_{O F F}=e^{-\alpha t_{O F F}}\left[T_{M I N}\left(\theta+t_{O N}\right)-T_{O F F}\right] .
\end{aligned}
$$

It is useful for the subsequent analysis to keep track of the $k$-th maximum (and possibly minimum) point of $T(t)$ (since initial time $t=0$ ) by defining variables $T^{+}(k)$ and $T^{-}(k)$ to be the time instant at which maximum and minimum temperature values are assumed for the $k$-th time respectively. In particular, there exists a function $F: \mathbb{R} \rightarrow \mathbb{R}$ such that:

$$
T^{+}(k+1)=F\left(T^{+}(k)\right) .
$$


Notice that due to continuity of $T_{M I N}$ and $T_{M A X}$ with respect to $t$ and continuity of solutions, $F$ is also continuous. To obtain a more explicit expression for $F$ we may define the following functions:

$$
\begin{aligned}
f_{M A X}^{O F F}(t) & =t+\frac{1}{\alpha} \log \left(T_{O F F}-T_{M A X}(t)\right) \\
f_{M I N}^{O F F}(t) & =t+\frac{1}{\alpha} \log \left(T_{O F F}-T_{M I N}(t)\right) \\
f_{M A X}^{O N}(t) & =t+\frac{1}{\alpha} \log \left(T_{M A X}(t)-T_{O N}\right) \\
f_{M I N}^{O N}(t) & =t+\frac{1}{\alpha} \log \left(T_{M I N}(t)-T_{O N}\right) .
\end{aligned}
$$

Assume that $T^{+}(1)=0$. By explicit integration of (26) we obtain:

$$
\begin{aligned}
f_{M I N}^{O N}\left(T^{-}(k)\right) & =f_{M A X}^{O N}\left(T^{+}(k)\right) \\
f_{M A X}^{O F F}\left(T^{+}(k+1)\right) & =f_{M I N}^{O F F}\left(T^{-}(k)\right) .
\end{aligned}
$$

Notice that whenever:

$$
\begin{aligned}
-\alpha\left[T_{M I N}(t)-T_{O N}\right] & <T_{M I N}^{\prime}(t) \\
T_{M A X}^{\prime}(t) & <\alpha\left[T_{O F F}-T_{M A X}(t)\right]
\end{aligned}
$$

the functions $f_{M A X}^{O F F}$ and $f_{M I N}^{O N}$ are monotone with respect to $t$ so that their inverse is well defined and one may define the recursion:

$$
T^{+}(k+1)=f_{M A X}^{O F F^{-1}}\left(f_{M I N}^{O F F}\left(f_{M I N}^{O N}{ }^{-1}\left(f_{M A X}^{O N}\left(T^{+}(k)\right)\right)\right)\right)=F\left(T^{+}(k)\right) \quad k=1,2, \ldots
$$

Notice that the functions $f_{M}^{O}$ with $\mathrm{M}=\mathrm{MIN}, \mathrm{MAX}$ and $\mathrm{O}=\mathrm{ON}, \mathrm{OFF}$, fulfill the following property:

$$
f_{M}^{O}(t \pm \Upsilon)=f_{M}^{O}(t) \pm \Upsilon \quad \forall t \in \mathbb{R}
$$


Similarly, for inverse functions we have:

$$
f_{M}^{O^{-1}}(t \pm \Upsilon)=f_{M}^{O-1}(t) \pm \Upsilon
$$

Exploiting the properties in (35) and (36) we rewrite recursion (34) in terms of the new variable $\varphi$ defined as:

$$
\varphi(k)=T^{+}(k)-k \Upsilon .
$$

The variable $\varphi(k)$ keeps track of the phase difference (expressed in time units) between temperature peaks and the forcing periodic signals $T_{M I N}(t)$ and $T_{M A X}(t)$. Straightforward manipulations show that letting

$$
F(t)=f_{M A X}^{O F F^{-1}}\left(f_{M I N}^{O F F}\left(f_{M I N}^{O N}{ }^{-1}\left(f_{M A X}^{O N}(t)\right)\right)\right)
$$

we have the equation:

$$
\begin{aligned}
\varphi(k+1) & =T^{+}(k+1)-(k+1) \Upsilon=F\left(T^{+}(k)\right)-(k+1) \Upsilon \\
& =F\left(T^{+}(k)-k \Upsilon\right)-\Upsilon=F(\varphi(k))-\Upsilon
\end{aligned}
$$

Three distinct scenarios are possible for the recursion:

$$
\varphi(k+1)=F(\varphi(k))-\Upsilon
$$

1. The first possibility is that $F(\varphi)-\Upsilon>\varphi$ for all $\varphi \in \mathbb{R}$. In this case $\varphi(k)$ is an increasing sequence (with positive average slope). This implies that temperature oscillations are slowlier than those of the forcing signal.

2. The second possibility is that $F(\varphi)-\Upsilon<\varphi$ for all $\varphi \in \mathbb{R}$. In this case $\varphi(k)$ is a decreasing sequence (with negative average slope). In this case temperature 


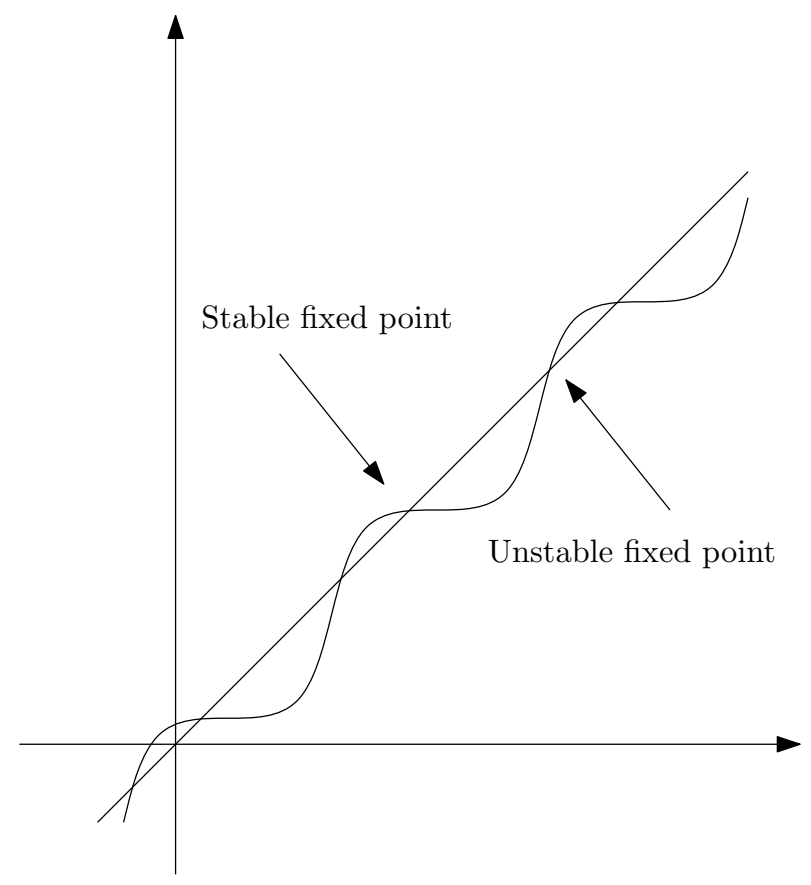

Figure 9: Stable and unstable fixed points of (38)

oscillations are faster than those of the forcing signal.

3. The last possibility is that there exist fixed points of the map $F(\varphi)-\Upsilon$ (in particular if $\bar{t}$ is a fixed point than also $\bar{t} \pm n \Upsilon$ is a fixed point for all $n \in \mathbb{N}$ ). This happens whenever:

$$
\Upsilon \in\left(\hat{\tau}_{M I N}, \hat{\tau}_{M A X}\right)
$$

Stability of fixed points can be easily determined by checking the relative slopes of intersection points of the map $F(\varphi)-\Upsilon$ with the diagonal (see Fig. 9).

If the function $F(\varphi)-\Upsilon$ only crosses twice the diagonal on each interval of length $\Upsilon$, then only two fixed points will exist (modulo equivalence $\pm n \Upsilon$ ), one almost globally asymptotically stable and the other unstable. From a physical point of view this situation corresponds to a scenario in which phase synchronization occurs and temperature oscillations happen at the same frequency of the forcing signal. 
Notice that, in a closed-loop scenario in which $T_{M I N}(t)$ and $T_{M A X}(t)$ are determined as a function of the global network frequency, and frequency fluctuations are in turn determined by variations in the absorbed power which is periodic of period $\tau$, necessarily $\tau=\Upsilon$. That is, scenario 1 and 2 are not possible in a periodic regime in which deterministic hysteretic regulation is adopted. The above analysis suggests that the presence of small periodic ripples in networks frequency will gradually entrain oscillations of fridges that have nearby oscillations frequency, thus reinforcing the frequency ripple and eventually lead to even larger numbers of entrained refrigerators. Simulations indeed confirm the intrinsic risks of such regulation approach as catastrophic oscillations eventually develop in the network. 